\title{
Paraoxonase Activity and Oxidative Status in Patients with Tinnitus
}

\author{
Sema Koç ${ }^{1}$, Servet Akyüz ${ }^{2}$, Battal Tahsin Somuk ${ }^{2}$, Harun Soyalic ${ }^{2}$, Beyhan Yılmaz ${ }^{3}$, \\ Abdullah Taskin ${ }^{4}$, Hasan Bilinc ${ }^{4}$, and Nurten Aksoy ${ }^{4}$ \\ 1 Department of Otorhinolaryngology, Antalya Education and Research Hospital, Antalya, \\ 2Department of Otorhinolaryngology, Gaziosmanpasa University School of Medicine, Tokat, \\ ${ }^{3}$ Department of Otorhinolaryngology, Dicle University School of Medicine, Diyarbakır, \\ ${ }^{4}$ Department of Biochemistry, Harran University School of Medicine, Sanliurfa, Turkey
}

Received October 2, 2015

Revised December 17, 2015

Accepted January 21, 2016

\section{Address for correspondence \\ Sema Koç, MD \\ Department of Otorhinolaryngology, \\ Antalya Education and \\ Research Hospital, \\ Kazim, Karebekir St \\ Antalya 07100, Turkey \\ Tel +905054983511 \\ Fax +902422496302 \\ E-mail drsemakoc@gmail.com}

Background and Objectives: The aim of this study was to investigate serum paraoxanase-1 (PON) activity, total oxidant status (TOS), total antioxidant status (TAS), and the oxidative stress index (OSI) in tinnitus; and to compare the results with data from healthy subjects. Subjects and Methods: A total of 114 subjects- 54 patients with tinnitus and 60 healthy controls were enrolled in this study. Serum PON activity, TOS, TAS, and OSI levels were measured. Results: In the tinnitus group, TAS, and PON were significantly lower than in the control group $(p<0.001)$. However, the TOS, and OSI levels were significantly higher in the tinnitus group than in the control group $(p<0.001)$. Conclusions: According to the data obtained from the present study, patients with tinnitus were exposed to potent oxidative stress. Oxidative stress may be the key contributing factor to the pathogenesis of tinnitus.

J Audiol Otol 2016;20(1):17-21

KEY WORDS: Tinnitus · Paraoxonase · Total oxidant status · Total antioxidant status . Oxidative stress index.

\section{Introduction}

Tinnitus is defined as subjective perception of noises in the absence of an external auditory stimulus [1]. Epidemiological investigations demonstrate that $10-15 \%$ of the general population is affected by tinnitus [2]. Age and hearing impairment increase the prevalence of tinnitus. The cause of tinnitus remains unclear, although a broad variety of factors have been considered to explain the etiology and pathogenesis of the development of tinnitus [3]. Acoustic trauma, stress, ototoxic drugs, high doses of salicylate, sudden hearing loss, some otological and neurological diseases, such as Meniere's disease, acoustic neuroma, otosclerosis and vascular compression, are factors that may contribute to the pathogenesis of tinnitus.

This is an Open Access article distributed under the terms of the Creative Commons Attribution Non-Commercial License (http://creativecommons. org/licenses/by-nc/3.0/) which permits unrestricted non-commercial use, distribution, and reproduction in any medium, provided the original work is properly cited.
It has been recently observed that reactive oxygen species (ROS) plays a role in the pathogenesis of otological and neurological diseases [4-8].

Paraoxonase-1 (PON), total oxidant status (TOS), total antioxidant status (TAS), and oxidative stress index (OSI) are studied as oxidative stress markers for human. In general, increased the free radical production above the normal threshold leads to exhaustion and thus reduction in antioxidant status in biological system. PON is a 354 amino acid protein synthesized mainly in the liver and secreted into the blood PON is a high-density lipoprotein (HDL)-associated antioxidant enzyme that protects low-density lipoprotein (LDL) from oxidation by hydrolyzing lipid peroxides in oxidized lipoproteins [9].

The aim of this study was to investigate the serum PON activity as well as to determine oxidative status via measurement of TOS, TAS, and OSI in the tinnitus and to compare these results to data from healthy subjects. 


\section{Subjects and Methods}

\section{Subjects}

This is a prospective, controlled study of patients with tinnitus. The study was approved by the local ethics committee. Patient consents were obtained before the procedures. A total of 114 subjects-54 patients with tinnitus and 60 healthy controls were enrolled in this study. All patients underwent a baseline evaluation including a detailed medical history, typical otorhinolaryngologic and audiological evaluation (pure tone, speech, and admittance audiometry, transient otoacoustic emission and acoustic reflex tests), full neuro-otological diagnostics (direct tests that observe static and dynamic balance, evidence of coordination, and function of the vestibularocular reflex through head impulse, shifting of the line of gaze, and spontaneous and semi-spontaneous nystagmus observations) and blood tests. The main inclusion criteria were being between 18 and 65 years of age, the presence of continuous tinnitus with a duration of $>1$ year, chronic subjective cochlear tinnitus, and no treatment of tinnitus within 8 weeks prior to entry into the study. Otorhinolaryngologic, neurootological examinations and audiological evaluation were normal for all study participants. The exclusion criteria were the presence of one or more of the following: any pathologic conditions of the ear, nose, and throat that might be responsible for tinnitus, such as middle or external ear problems, otosclerosis, chronic otitis media, Meniere's disease, vestibular schwannoma, venous hum and vascular neoplasm. Patients with a clinical history of ear and neuro-otologic surgery, taking ototoxic drugs, with temporal bone trauma, liver, hematological, cardiovascular, metabolic, neurologic, or psychiatric diseases and with malignancy were also excluded. After overnight fasting, peripheral venous blood samples were taken from patients with tinnitus and healthy volunteers into empty tubes for one time only. Serum PON activity, TOS, TAS, and OSI were measured.

\section{Blood samples collection}

Fasting peripheral venous blood samples were immediately centrifuged at $3000 \times \mathrm{g}$ for $10 \mathrm{~min}$, then stored at $-80^{\circ} \mathrm{C}$ until they were needed for further analysis of PON activity along with determination of oxidative status via measurement of TOS, TAS, and OSI.

\section{Measurement of paraoxonase activity}

Paraoxonase activity was measured in the absence (basal activity) and presence of $\mathrm{NaCl}$ (salt-stimulated activity). Briefly, the rate of paraoxon hydrolysis was measured by an increase in absorbance at $412 \mathrm{~nm}$ at $25^{\circ} \mathrm{C}$. The amount of p-ni- trophenol generated was calculated from the molar absorptivity coefficient at $\mathrm{pH} 8$, which was $17.100 \mathrm{M}^{-1} \mathrm{~cm}^{-1}$. Paraoxonase activity was expressed as $\mathrm{U} / \mathrm{L}$ serum $[10,11]$.

\section{Measurement of TOS}

The TOS of the serum was determined using a novel automated measurement method [12]. Oxidants present in the sample oxidize the ferrous ion-o-dianisidine complex to ferric ion. This oxidation reaction is enhanced by glycerol molecules, which are abundantly present in the reaction medium. The ferric ion reacts with xylenol orange in an acidic medium to produce a colored complex. The intensity of color, which can be measured spectrophotometrically, is related to the total amount of oxidant molecules in the sample. The assay is calibrated with hydrogen peroxide and the results are expressed in terms of micromolar hydrogen peroxide equivalents per liter $\left(\mu \mathrm{mol} \mathrm{H}_{2} \mathrm{O}_{2}\right.$ equiv/L). The assay has excellent precision values that are lower than $2 \%$.

\section{Measurement of the TAS}

The TAS of the serum was determined using an automated measurement method [12]. Briefly, potent free radical reactions were initiated with the production of a hydroxyl radical via the Fenton reaction and the rate of reactions was monitored by measuring the absorbance of colored dianisidyl radicals. Using this method, the antioxidative effect of the sample against potent free radical reactions, which were initiated by synthesized hydroxyl radical, was measured. An automated analyzer (Aeroset $^{\circledR}$, Abbott, IL, USA) was used. Both intraand interassay coefficients of variations were lower than $3 \%$. The data were expressed as $\mu \mathrm{mol}$ Trolox Equiv/L.

\section{Statistical analysis}

Pearson's chi-square test was used to compare the gender between groups. Gender was presented as count and percentage. The Kolmogorov-Smirnov test was used to evaluate whether the variables were normally distributed. The two independent sample $t$ test or Mann Whitney $U$ test were used to compare continuous variables between control and patient groups. Continuous variables were presented as mean (standard deviation) or median [interquartile range (Q1-Q3)]. SPSS software 15.0 for Windows (SPSS Inc., Chicago, IL, USA) was used for all statistical analysis. $p$-values were considered statistically significant when they were $<0.05$.

\section{Results}

The tinnitus group consisted of 54 patients, 29 male (54\%), between the ages of 17 and 65 years (mean age, $48 \pm 12$ ) and 
Table 1. Study parameters in the tinnitus and control groups

\begin{tabular}{lccc}
\hline & Tinnitus group $(n=54)$ & Control group $(n=60)$ & $p$ \\
\hline PON, $U / L^{*}$ & $138 \pm 37$ & $169 \pm 43$ & $<0.001$ \\
TOS, $\mu$ mol $\mathrm{H}_{2} \mathrm{O}_{2}$ equiv/L ${ }^{+}$ & $15.9(13.7-21)$ & $11.6(10.3-13.6)$ & $<0.001$ \\
TAS, $\mu$ mol Trolox Equiv/L* & $1.00 \pm 0.12$ & $1.12 \pm 0.13$ & $<0.001$ \\
OSI, arbitrary units* & $1.77 \pm 0.48$ & $1.08 \pm 0.25$ & $<0.001$ \\
\hline
\end{tabular}

*values are presented as mean \pm standard deviation, ${ }^{\dagger}$ values are presented as median (Q1-Q3). PON: paraoxanase-1, TOS: total oxidant status, TAS: total antioxidant status, OSI: oxidative stress index

the control group consisted of 60 individuals, 25 male (42\%) between the ages of 18 and 65 (mean age, $45 \pm 14$ ). There were no statistical differences in either the age and gender between the tinnitus and control groups.

In the tinnitus group, TAS and PON were significantly lower than the control group (TAS: $1.00 \pm 0.12 \mu \mathrm{mol}$ Trolox Equiv/L vs. $1.12 \pm 0.13 \mu \mathrm{mol}$ Trolox Equiv/L, $p<0.001$ and PON: $138 \pm 37 \mathrm{U} / \mathrm{L}$ vs. $169 \pm 43 \mathrm{U} / \mathrm{L}$, respectively, $p<0.001$ ). However, the TOS and OSI levels were significantly higher in the tinnitus group than in the control group (TOS: 15.9 (13.721) $\mu \mathrm{mol} \mathrm{H}_{2} \mathrm{O}_{2}$ equiv/L vs. 11.6 (10.3-13.6) $\mu \mathrm{mol} \mathrm{H}_{2} \mathrm{O}_{2}$ equiv/L, $p<0.001$ and OSI: $1.77 \pm 0.48$ arbitrary units vs. $1.08 \pm$ 0.25 arbitrary units, $p<0.001$, respectively). Table 1 summarizes the statistical results.

\section{Discussion}

In order to evaluate the correlation between tinnitus and oxidative stress, we measured PON, TOS, TAS, and OSI. To the best of our knowledge, there is no study reported in the literature related to PON, TOS, TAS, and OSI in patients with tinnitus.

ROS including superoxide radical $\left(\mathrm{O}_{2}^{-}\right)$, hydroxyl radical $\left(\mathrm{OH}^{-}\right)$, and hydrogen peroxide $\left(\mathrm{H}_{2} \mathrm{O}_{2}\right)$, are generated in the cochlea during normal aerobic metabolism in cells at physiological concentrations. There are defense systems that protect against ROS damage that involve enzymatic and non-enzymatic antioxidant systems. The enzymatic systems include superoxide dismutase, glutathione peroxidase, glutathione reductase, and catalase. On the other hand, the non-enzymatic systems include various substances like vitamin $\mathrm{E}$, vitamin $\mathrm{C}$, beta-carotene, glutathione, uric acid, bilirubin, and retinol [13-15]. The ROS production and removal rates are in balance, and this situation is called oxidative balance. As long as oxidative balance is maintained, the organism is not affected by the ROS. Oxidant production can take place at an excessive rate, a condition known as oxidative stress, and it may be the result of an increase in ROS and/or impairment in the antioxidant mechanism [16]. ROS can majorly affect cells by damaging lipids and destroying the cell membrane, by inactivating enzymes and thus affecting protein production, by depolymerizing polysaccharides and thus damaging carbohydrates, by damaging, mitochondrial DNA and causing mitochondrial dysfunction [17]. High concentrations of ROS can have cytotoxic and neurotoxic effects, resulting in damage to the auditory hair cells of the labyrinth and the acoustic system. The role of oxidative stress in certain inner ear disorders, such as Meniere's disease, noise-induced hearing loss, labyrinthitis, cisplatin ototoxicity and aminoglycoside ototoxicity is well known $[18,19]$. The etiopathogenesis of tinnitus is not yet clearly defined. In most patients, the development of tinnitus can be linked to damage to the cochlea [20]. Experimental studies have indicated that ROS can cause damage to the cochlear sensorial epithelium [21]. Neri, et al. [22] evaluated the oxidative stress of 44 patients with idiopathic tinnitus and 25 healthy volunteers. They reported that oxidative damage markers (malonaldehyde, 4-hydroxynonenal, myeloperoxidase) were higher in patients with tinnitus than in the control group, whereas glutathione peroxidase, an antioxidant enzyme, had lower activity in the tinnitus group. Based on these findings, they concluded that oxidative stress is important in the pathogenesis of tinnitus.

Lipid peroxidation, which is caused by ROS, is one of the most important causes of cell damage. The PON has paraoxonase, arylesterase, and dyazoxonase activities and is a particularly important antioxidant enzyme in the human body. This enzyme has a significant role in preventing LDL and HDL oxidation by hydrolyzing lipid peroxide products [9]. These enzymes may potentially involve in a great variety of oxidative damage associated with diseases. These include neurodegenerative diseases, such as Alzheimer and Parkinson diseases, cardiovascular diseases, chronic renal failure and metabolic syndrome. It has been demonstrated that noise induces the local release of ROS including superoxide radicals $\left(\mathrm{O}_{2}^{-}\right)$, hydroxyl radicals $\left(\mathrm{OH}^{-}\right)$, and hydrogen peroxide $\left(\mathrm{H}_{2} \mathrm{O}_{2}\right)$ that may lead to damage to the cochlear sensorineural epithelium [21-23]. Fortunato, et al. [24] reported that noise-induced hearing loss is related to a gene polymorphisms in PON and superoxide dismutase.

Levels of numerous oxidant and antioxidant parameters may be measured separately. Since oxidants and antioxidants have an additive impact, individual values may not accurate- 
ly indicate the TOS or TAS. Therefore, TOS and TAS are more correct indicators of the oxidative and antioxidative states of persons [25].

Tinnitus is a complex, multifactorial dissese which includes numerous etiologic loci. The major reason of tinnitus is injury to hearing sensory cells of the cochlea, with or without association to a damage of auditory cortex pathway related to biochemical changes, inflammation, and damages induced by ROS [26]. Under normal circumstances, the cochlea comprises particles containing antioxidant vitamins and enzymes. In cases of some inner ear disease, the certainly arising antioxidant systems may not reduce sufficient detoxifying effects and oxidative stress develops as a result of imbalance between ROS and scavenging of these reactants [16]. The sensorineural epithelium is at major risk of radical-induced lesions in the cochlea. There are some studies recommending that oxidative stress could damage the epithelium of the labyrinth and the acoustic and vestibular nervous system [16]. Levine [27] theorized that a decrease in cochlear nerve input leads to disinhibition of the dorsal cochlear nucleus and hyperactivity of the auditory cortex which is felt as tinnitus.

Tinnitus has significant clinical impact, a number of therapeutic approaches have been described, including herbal medicines, acupuncture, hearing aids, tinnitus masking, various drug treatments, tinnitus retraining. Some studies have shown favorable results, while others did not result in benefits. Various substances have been used and tested as drug treatments. Among them, antioxidants have appeared to be promising [28]. Savastano, et al. [17] reported that antioxidant therapy in patients with idiopathic tinnitus seems to reduce the subjective discomfort and tinnitus intensity. Polanski, et al. [28] described that there was no benefit from the use of antioxidant agents for tinnitus in their study. Gopal, et al. [29] showed that by means of objective and subjective assessments, tinnitus decreases following antioxidant therapy and they declared that antioxidant agents may be a pharmacological optionin in tinnitus management. Experimental educations on the cochlea have revealed that administrations of substances able to define oxidative stress can decrease neuromediators release, and that concomitant injection of antioxidants can decrease the bioelectric stimulations [22].

In our study, in the tinnitus group, the TAS level and PON activity were significantly lower than in the control group. However, the TOS and OSI levels were significantly higher in the tinnitus group than in the control group. In conclusion, according to the data obtained from the present study, patients with tinnitus are exposed to potent oxidative stress. Oxidative stress may be a key contributing factor in the pathogenesis of tinnitus. However, further studies with larger sample sizes are needed to define the exact role of oxidative stress in the pathogenesis and treatment of the disease.

\section{Conflicts of interest}

The authors have no financial conflicts of interest.

\section{REFERENCES}

1) Jufas NE, Wood R. The use of benzodiazepines for tinnitus: systematic review. J Laryngol Otol 2015;129 Suppl 3:S14-22.

2) Nemholt SS, Schmidt JH, Wedderkopp N, Baguley DM. Prevalence of tinnitus and/or hyperacusis in children and adolescents: study protocol for a systematic review. BMJ Open 2015;5:e006649.

3) Lee JH, Ra JJ, Kim YH. Adequacy of the simplified version of the Tinnitus Handicap Inventory (THI-S) to measure tinnitus handicap and relevant distress. Korean J Audiol 2014;18:19-27.

4) Karatas E, Deniz M. The comparison of acoustic and psychic parameters of subjective tinnitus. Eur Arch Otorhinolaryngol 2012;269: 441-7.

5) Calabrese V, Cornelius C, Maiolino L, Luca M, Chiaramonte R, Toscano MA, et al. Oxidative stress, redox homeostasis and cellular stress response in Ménière's disease: role of vitagenes. Neurochem Res 2010;35:2208-17.

6) Bonabi S, Caelers A, Monge A, Huber A, Bodmer D. Resveratrol protects auditory hair cells from gentamicin toxicity. Ear Nose Throat J 2008;87:570-3.

7) Terao K, Cureoglu S, Schachern PA, Morita N, Nomiya S, Deroee $\mathrm{AF}$, et al. Cochlear changes in presbycusis with tinnitus. Am J Otolaryngol 2011;32:215-20.

8) Goswami B, Tayal D, Gupta N, Mallika V. Paraoxonase: a multifaceted biomolecule. Clin Chim Acta 2009;410:1-12.

9) Metin ZB, Aydin S, Unur M, Cakmakoglu B, Toptas B, Hafiz G, et al. Oral squamous cell carcinoma and serum paraoxonase 1. J Laryngol Otol 2013;127:1208-13.

10) Eckerson HW, Wyte CM, La Du BN. The human serum paraoxonase/arylesterase polymorphism. Am J Hum Genet 1983;35:1126-38.

11) Erel O. A new automated colorimetric method for measuring total oxidant status. Clin Biochem 2005;38:1103-11.

12) Erel O. A novel automated method to measure total antioxidant response against potent free radical reactions. Clin Biochem 2004;37: 112-9.

13) Choi CH. Mechanisms and treatment of blast induced hearing loss. Korean J Audiol 2012;16:103-7.

14) Doğruer ZN, Unal M, Eskandari G, Pata YS, Akbaș Y, Cevik T, et al. Malondialdehyde and antioxidant enzymes in children with obstructive adenotonsillar hypertrophy. Clin Biochem 2004;37:718-21.

15) Seidman MD, Ahmad N, Joshi D, Seidman J, Thawani S, Quirk WS. Age-related hearing loss and its association with reactive oxygen species and mitochondrial DNA damage. Acta Otolaryngol Suppl 2004; (552):16-24.

16) Gilles A, Ihtijarevic B, Wouters K, Van de Heyning P. Using prophylactic antioxidants to prevent noise-induced hearing damage in young adults: a protocol for a double-blind, randomized controlled trial. Trials 2014;15:110.

17) Savastano M, Brescia G, Marioni G. Antioxidant therapy in idiopathic tinnitus: preliminary outcomes. Arch Med Res 2007;38:456-9.

18) Takumida M, Anniko M, Popa R, Zhang DM. Pharmacological models for inner ear therapy with emphasis on nitric oxide. Acta Otolaryngol 2001;121:16-20.

19) Sataloff RT, Bittermann T, Marks L, Lurie D, Hawkshaw M. The effects of glutathione enhancement on sensorineural hearing loss. Ear Nose Throat J 2010;89:422-33.

20) An YH, Jin SY, Yoon SW, Shim HJ. The effects of unilateral tinnitus on auditory temporal resolution: gaps-in-noise performance. Korean 
J Audiol 2014;18:119-25.

21) Park SY, Back SA, Kim HL, Kim DK, Yeo SW, Park SN. Renexin as a rescue regimen for noise-induced hearing loss. Noise Health 2014; 16:257-64.

22) Neri S, Signorelli S, Pulvirenti D, Mauceri B, Cilio D, Bordonaro F, et al. Oxidative stress, nitric oxide, endothelial dysfunction and tinnitus. Free Radic Res 2006;40:615-8.

23) Camps J, Marsillach J, Joven J. The paraoxonases: role in human diseases and methodological difficulties in measurement. Crit Rev Clin Lab Sci 2009;46:83-106.

24) Fortunato G, Marciano E, Zarrilli F, Mazzaccara C, Intrieri M, Calcagno G, et al. Paraoxonase and superoxide dismutase gene polymorphisms and noise-induced hearing loss. Clin Chem 2004;50: 2012-8.

25) Koc S, Aksoy N, Bilinc H, Duygu F, Uysal IÖ, Ekinci A. Paraox- onase and arylesterase activity and total oxidative/anti-oxidative status in patients with chronic adenotonsillitis. Int J Pediatr Otorhinolaryngol 2011;75:1364-7.

26) Nondahl DM, Cruickshanks KJ, Huang GH, Klein BE, Klein R, Nieto FJ, et al. Tinnitus and its risk factors in the Beaver Dam offspring study. Int J Audiol 2011;50:313-20.

27) Levine RA. Tinnitus: diagnostic approach leading to treatment. Semin Neurol 2013;33:256-69.

28) Polanski JF, Soares AD, de Mendonça Cruz OL. Antioxidant therapy in the elderly with tinnitus. Braz J Otorhinolaryngol 2015 Oct 17 [Epub ahead of print]. http://dx.doi.org/10.1016/j.bjorl.2015.04.016.

29) Gopal KV, Thomas BP, Mao D, Lu H. Efficacy of carnitine in treatment of tinnitus: evidence from audiological and MRI measures-a case study. J Am Acad Audiol 2015;26:311-24. 